\title{
A!
}

This is an electronic reprint of the original article.

This reprint may differ from the original in pagination and typographic detail.

Herranen, Touko; Laurson, Lasse

\section{Bloch-line dynamics within moving domain walls in 3D ferromagnets}

Published in:

Physical Review B

DOI:

10.1103/PhysRevB.96.144422

Published: 18/10/2017

Document Version

Publisher's PDF, also known as Version of record

Please cite the original version:

Herranen, T., \& Laurson, L. (2017). Bloch-line dynamics within moving domain walls in 3D ferromagnets.

Physical Review B, 96(14), [144422]. https://doi.org/10.1103/PhysRevB.96.144422

This material is protected by copyright and other intellectual property rights, and duplication or sale of all or part of any of the repository collections is not permitted, except that material may be duplicated by you for your research use or educational purposes in electronic or print form. You must obtain permission for any other use. Electronic or print copies may not be offered, whether for sale or otherwise to anyone who is not an authorised user. 


\title{
Bloch-line dynamics within moving domain walls in 3D ferromagnets
}

\author{
Touko Herranen* and Lasse Laurson \\ COMP Centre of Excellence and Helsinki Institute of Physics, Department of Applied Physics, Aalto University, P.O. Box 11100, \\ FI-00076 Aalto, Espoo, Finland
}

(Received 16 May 2017; revised manuscript received 30 August 2017; published 18 October 2017)

\begin{abstract}
We study field-driven magnetic domain wall dynamics in garnet strips by large-scale three-dimensional micromagnetic simulations. The domain wall propagation velocity as a function of the applied field exhibits a low-field linear part terminated by a sudden velocity drop at a threshold field magnitude, related to the onset of excitations of internal degrees of freedom of the domain wall magnetization. By considering a wide range of strip thicknesses from $30 \mathrm{~nm}$ to $1.89 \mu \mathrm{m}$, we find a nonmonotonic thickness dependence of the threshold field for the onset of this instability, proceeding via nucleation and propagation of Bloch lines within the domain wall. We identify a critical strip thickness above which the velocity drop is due to nucleation of horizontal Bloch lines, while for thinner strips and depending on the boundary conditions employed, either generation of vertical Bloch lines, or close-to-uniform precession of the domain wall internal magnetization takes place. For strips of intermediate thicknesses, the vertical Bloch lines assume a deformed structure due to demagnetizing fields at the strip surfaces, breaking the symmetry between the top and bottom faces of the strip, and resulting in circulating Bloch line dynamics along the perimeter of the domain wall.
\end{abstract}

DOI: 10.1103/PhysRevB.96.144422

\section{INTRODUCTION}

Dynamics of driven domain walls (DWs) in ferromagnets of various confined geometries such as wires, strips, and thin films is an important problem both from the pointof-view of technological applications, as well as due to strong fundamental physics interests. One central feature of DW dynamics driven by applied magnetic fields [1-4] or spin-polarized electric currents [5-10] is that the relation between the driving force (i.e., field or current density) and the resulting DW velocity tends to be nonmonotonic: In addition to contributing to DW propagation, a sufficiently strong driving force may excite internal degrees of freedom of the domain wall, resulting in an abrupt drop in the force-velocity curve of the DW. Only in some special cases such as when considering cylindrical nanowires, such a breakdown may be avoided [11]. Within the one-dimensional (1D) model [12], these internal degrees of freedom are described by an angle corresponding to the orientation of the DW internal magnetization, which starts precessing above the Walker field or current density, resulting in an abrupt drop in the DW propagation velocity.

While the above description in terms of the 1D model should apply for narrow nanowires and nanostrips, the situation is more complex when DWs in strips with a non-negligible width and/or thickness are considered. There, the excitation of the DW internal degrees of freedom, taking place concurrently with a drop in the DW propagation velocity, may be spatially nonuniform, and thus cannot be described by a single angular variable. In particular, in sufficiently wide thin ferromagnetic strips with perpendicular magnetic anisotropy (PMA), the velocity drop takes place via nucleation and subsequent propagation along the domain wall of vertical Bloch lines (VBLs) [12,13], i.e., transition regions separating different chiralities of the Bloch DW along its long axis. For thick enough strips or films with PMA, another type of excitation

*touko.herranen@aalto.fi is expected to become prominent, namely the nucleation of horizontal Bloch lines (HBLs) [12]; there, the DW chirality changes when moving along the DW in the thickness direction of the sample. These Bloch line structures, in particular in various garnet films, have been intensively studied already in the 1970s as they were at the time seen as potential building blocks of novel types of memory devices, the magnetic bubble memories [14-17]. Notice that a somewhat analogous scenario occurs in strips of in-plane magnetized materials such as permalloy, where a similar velocity drop takes place due to repeated nucleation and propagation of (anti)vortices across the strip width $[18,19]$.

Many of the related key studies of bubble materials such as garnet films where the presence of Bloch lines is essential for DW dynamics consist of theoretical work coupled with experimental observations [20-23]. Due to recent advances in numerical techniques and the available computing power, it is now possible to perform full micromagnetic simulations of 3D samples with linear sizes reaching several microns, thus approaching the thickness range of typical garnet films studied in the past. In such simulations one may monitor the full 3D dynamics of the system and thus obtain a more complete picture of the DW dynamics as compared to the typical experiments where one could observe only the surface of the relatively thick film.

Thus, we perform here full 3D micromagnetic simulations of field-driven DW dynamics in garnet strips with a wide range of strip thicknesses, considering as an example material the $(\mathrm{GdTmPrBi})_{3}(\mathrm{FeGa})_{5} \mathrm{O}_{12}$ magnetic garnet [23]. Such materials may be grown epitaxially on a substrate, inducing a crystalline PMA [12], independent of the film/strip thickness, making it possible to systematically study the thickness dependence of the DW dynamics. For both periodic and open boundary conditions along the strip width, we find a low-field constant DW mobility regime, terminated at a sudden velocity drop at a threshold field magnitude. The threshold field and the corresponding (local) maximum of the DW propagation velocity exhibit a nonmonotonic dependence on the sample 
thickness, with a peak of the maximum velocity occurring in films of a thickness related to the HBL width. We investigate in detail the related excitations of the DW internal structure and find that for thin strips with open boundary conditions at the strip edges, the velocity drop is due to a VBL being nucleated from one of the strip edges, followed by its repeated propagation along the DW across the strip width. In contrast, the corresponding instability in thin samples with periodic boundary conditions proceeds via spatially close-to-uniform precession of the DW internal magnetization. In samples of intermediate thickness, the VBL structure is deformed due to the flux-closing tendency at the sample surfaces. This deformation results in interesting dynamics where the high spin rotation part of the deformed VBL repeatedly rotates around the strip along the edges and surfaces of the strip, thus breaking the symmetry between the top and bottom strip surfaces. For the thickest films considered (up to $1.89 \mu \mathrm{m}$ ), the velocity drop is related to a HBL being nucleated from one of the sample surfaces, which subsequently moves back and forth along the DW in the thickness direction of the sample.

The paper is organized as follows: In Sec. II, we describe the details of our micromagnetic simulations, including material parameters used, and the geometry of the samples considered. In Sec. III, we study the DW velocity $v$ as a function of the driving field strength $B_{\text {ext }}$, sample thickness $h$ for the different boundary conditions, focusing in particular on the different excitation modes responsible for the velocity drop in the $v\left(B_{\text {ext }}\right)$ curves. In Sec. IV, we finish the paper by presenting our conclusions.

\section{METHODS}

The micromagnetic simulations are performed using the GPU-accelerated micromagnetic code MuMax3 [24-26], which solves numerically the Landau-Lifshitz-Gilbert equation $[27,28]$,

$$
\partial \mathbf{m} / \partial t=\gamma \mathbf{H}_{\text {eff }} \times \mathbf{m}+\alpha \mathbf{m} \times \partial \mathbf{m} / \partial t,
$$

where $\mathbf{m}=\mathbf{M} / M_{\mathrm{S}}$ is the magnetization, $\gamma$ the gyromagnetic ratio, $\alpha$ the Gilbert damping parameter, and $\mathbf{H}_{\text {eff }}$ the effective field, with contributions due to exchange, anisotropy, Zeeman, and demagnetizing energies.

We simulate DW dynamics in garnet strips with a wide range of thicknesses. As a test material we choose to consider $(\mathrm{GdTmPrBi})_{3}(\mathrm{FeGa})_{5} \mathrm{O}_{12}$ magnetic garnet with saturation magnetization $M_{\mathrm{S}}=8992 \mathrm{~A} / \mathrm{m}$, uniaxial out-of-plane anisotropy constant $K_{\mathrm{u}}=602.5 \mathrm{~J} / \mathrm{m}^{3}$, exchange constant $A=2.2 \times 10^{-12} \mathrm{~J} / \mathrm{m}$, and damping parameter $\alpha=0.15$ [23]. The quality factor for this material is $Q=K_{\mathrm{u}} / K_{\mathrm{d}}=11.9$, with the stray field energy constant given by $K_{\mathrm{d}}=M_{\mathrm{S}}{ }^{2} \mu_{0} / 2 \approx$ $50.8 \mathrm{~J} / \mathrm{m}^{3}$, where $\mu_{0}$ is vacuum permeability. The Bloch wall width parameter $\Delta=\sqrt{A / K_{\mathrm{u}}} \approx 60 \mathrm{~nm}$.

In the simulations, we fix the sample width to $w=3.84 \mu \mathrm{m}$ and use a moving simulation window of length $30.72 \mu \mathrm{m}$ centered around the DW; during the simulation the simulation window is shifted such that the average DW $x$ position is always kept within one discretization cell from the middle of the sample in the $x$ direction. The thickness of the sample is varied between $h=30 \mathrm{~nm}$ and $h=1.89 \mu \mathrm{m}$. We use cubic

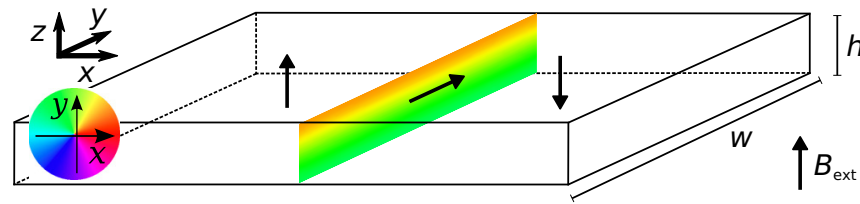

FIG. 1. Relaxed initial magnetization configuration for $B_{\mathrm{ext}}=0$ with a Bloch DW in the middle of a strip of thickness $h=990 \mathrm{~nm}$, separating the $\pm z$ magnetized domains. The arrows indicate the magnetization direction. Due to the demagnetizing fields acting on the DW and originating from the surface charges, the in-plane magnetization of the DW (visualized by the different colors as indicated by the color wheel) at the strip surfaces tilts from the $+y$ direction (light green) approximately by $37^{\circ}$ towards the $\pm x$ direction (orange and darker green, respectively) for this particular $h$ value, while the pure Bloch wall structure (DW magnetization along $+y$ ) is maintained for $z=h / 2$.

discretization cells with a side length of $\Delta_{\text {cell }}=30 \mathrm{~nm} \approx$ $\Delta / 2$ [29].

The magnetization is initialized into two domains, with magnetization along the $+z$ and $-z$ directions, respectively, with $\mathrm{a}+y$ magnetized Bloch wall separating the domains in the middle of the sample. The system is then let to relax to its equilibrium configuration. A relaxed micromagnetic configuration for $h=990 \mathrm{~nm}$ is presented schematically in Fig. 1. The domains generate magnetic surface charges, which in turn create in-plane demagnetizing fields acting on the DW magnetization at the sample surfaces. In a static $\left(B_{\text {ext }}=0\right)$ isolated DW, the demagnetizing field tends to tilt the DW magnetization towards a Néel structure close to the top and bottom surfaces, in order to close the flux $[12,30]$. The center of the wall remains in a pure Bloch wall structure. Theoretical calculations by Slonczewski show that the demagnetizing field component perpendicular to the wall in an isolated DW is $H_{S}(z)=4 M_{S} \ln [z /(h-z)]$ in the limit $\Delta / h=0$ [30], with the sample surfaces at $z=\{0, h\}$. Using this expression, one may define the so-called critical points where $\left|H_{S}(z)\right|=$ $8 M_{S}$, located at $z_{a}(h)=h /\left(1+e^{2}\right)$ and $z_{b}(h)=h e^{2} /\left(1+e^{2}\right)$, respectively [30]. These are understood as points where HBLs may be nucleated. The twist of the DW magnetization due to demagnetizing fields is suppressed by the exchange stiffness for thin samples [31].

In what follows we present an extensive micromagnetic study of the DW dynamics induced by driving fields $B_{\text {ext }}$ of different magnitudes along the $+z$ direction, by varying the boundary conditions (open vs periodic along the strip width) and the sample thickness $h$. More specifically, we address the question of how the dynamics of the internal magnetization of the DW affects the DW propagation velocity, focusing in particular on the excitations responsible for the sudden velocity drops in the $v\left(B_{\text {ext }}\right)$ curves.

\section{RESULTS}

To understand the details of field-driven domain wall dynamics in garnet strips of different thicknesses, we consider here separately two different boundary conditions along the $y$ direction (see Fig. 1), i.e., periodic boundary conditions (PBCs) mimicking an infinitely long DW and open boundary 

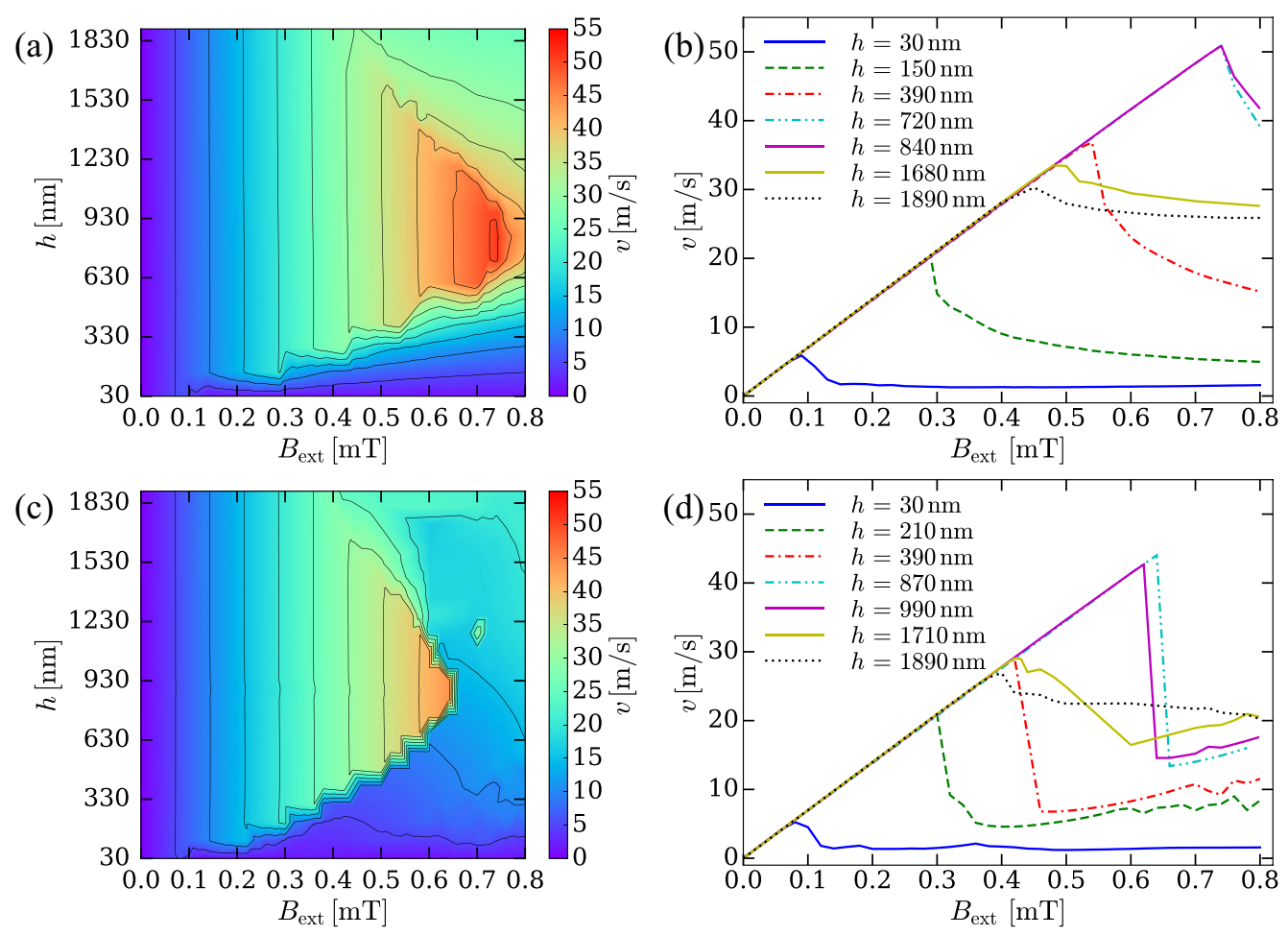

FIG. 2. Average field-driven DW propagation velocities $v$ as a function of $B_{\text {ext }}$ and $h$ for PBCs [(a) and (b)] and OBCs [(c) and (d)] in the $y$ direction. In each case, the velocities are calculated from the slope of a linear fit to the DW position $x(t)$, excluding the initial transient. For $B_{\text {ext }}>B_{\text {ext }}^{\max }(h)$, the transient time is estimated to be the time when the initially positive $y$ component of the DW magnetization first changes sign (indicating the onset of periodic precessional dynamics), while for smaller fields a fixed $100 \mathrm{~ns}$ transient time is removed from the start of each simulation run. The contour lines in (a) and (c) correspond to $5 \mathrm{~m} / \mathrm{s}$ difference in velocity.

conditions (OBCs) where the effects due to strip edges are important. Moreover, one should notice that in the case of OBCs, the DW has more freedom to respond to applied fields, e.g., by exhibiting global DW tilting [13], something that is excluded by construction when using PBCs where the "end points" of the DW are attached to each other. In both cases, we measure the relation between the DW propagation velocity $v$ and the applied field $B_{\text {ext }}$ along the $+z$ direction, i.e., $v\left(B_{\text {ext }}\right)$, and focus on the excitations of the DW internal magnetization responsible for the sudden drop of $v\left(B_{\text {ext }}\right)$ at a threshold field strength and how their nature depends on the boundary conditions and the sample thickness $h$. We consider fields up to $B_{\text {ext }}=0.8 \mathrm{mT}$, enough to observe the velocity breakdown in all the systems considered.

\section{A. Periodic boundary conditions}

We start by considering PBCs in the $y$ direction. The resulting DW propagation velocities as a function of $B_{\text {ext }}$ and $h$ are presented in Figs. 2(a) and 2(b). For $B_{\text {ext }}$ below the $h$-dependent threshold field (also referred to as the critical field in what follows) for the onset of the instability resulting in a velocity drop, $v\left(B_{\text {ext }}\right)$ is linear in $B_{\text {ext }}$. Within this linear regime, the DW magnetization remains constant during DW motion after an initial transient, and the DW mobility $\mu=\mathrm{d} v / \mathrm{d} B_{\text {ext }}$ is well described by the well-known theoretical result of $\mu_{\text {steady }}=\gamma \Delta / \alpha \approx 71 \mathrm{~ms}^{-1} \mathrm{mT}^{-1}$ for all $h$ [12].

The $h$-dependent peak DW propagation velocities $v_{\max }$, extracted from data in Fig. 2 considering PBCs along $y$, are presented in Fig. 3 (blue circles). The corresponding threshold fields for the onset of the instability, i.e., the fields $B_{\text {ext }}^{\max }$ at which the (local) maximum velocity $v_{\max }$ occurs, are reported in the inset of Fig. 3 as a function of $h$. Both $v_{\max }$ and $B_{\text {ext }}^{\max }$ display an intriguing nonmonotonic dependence on $h$. To understand this, we need to consider the different excitation

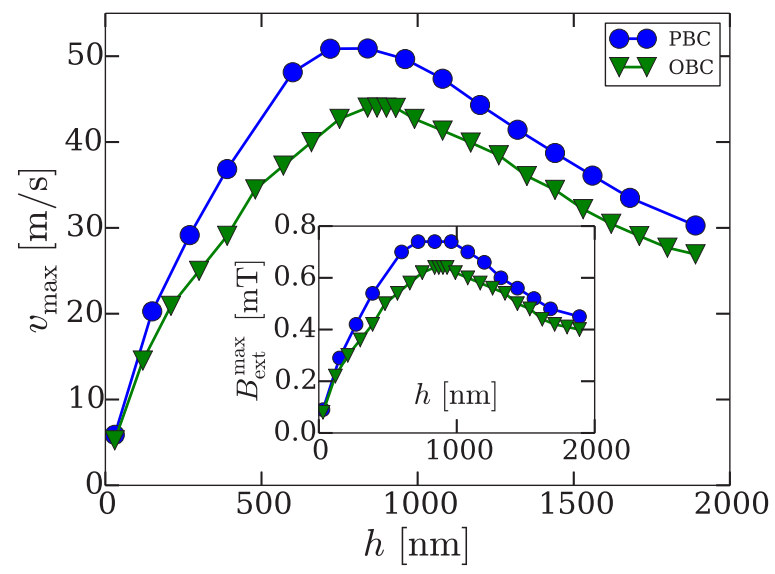

FIG. 3. Maximum of the average field-driven DW propagation velocity $v_{\max }$ before the velocity drop as a function of the sample thickness $h$. The inset depicts the $h$ dependence of the corresponding applied field value $B_{\mathrm{ext}}^{\max }$. OBCs in the $y$ direction give rise to lower values of $v_{\max }$ (and $B_{\mathrm{ext}}^{\max }$ ), given the additional excitation modes available due to open boundaries at the strip edges. 
modes of the DW internal magnetization responsible for the onset of the velocity drop and how these depend on $h$.

Thus, we consider next the dynamics of the DW internal magnetization just above the $h$-dependent critical field $B_{\text {ext }}^{\max }$ corresponding to the local maximum of the DW propagation velocity. In thin samples ( $h \leqslant 150 \mathrm{~nm}$ ), we observe spatially close-to-uniform rotation of the DW magnetization when fields just above the critical field are applied (see Supplemental Material, Movie 1 [32]), similarly to the Walker breakdown dynamics observed in nanowire geometries, and as predicted by the $1 \mathrm{D}$ model. As is visible in Movie 1, the spatially uniform DW magnetization rotation does not occur at a constant rate. The rotation is slowed down when the DW magnetization $m_{\text {DW }}$ assumes a tilted (due to the finite $B_{\text {ext }}$ ) Bloch wall configuration. After $B_{\text {ext }}$ has slowly rotated $m_{\mathrm{DW}}$ from the tilted Bloch towards a Néel configuration, $m_{\mathrm{DW}}$ rotates abruptly to reach the opposite tilted Bloch wall configuration, where the magnetization rotation slows down again.

When $h$ is increased towards "intermediate" thicknesses $(150 \mathrm{~nm}<h<720 \mathrm{~nm})$, we start to see some internal structure developing in the DW magnetization during the magnetization precession process. More specifically, we observe a "partial" HBL (meaning that it does not exhibit a full $\pi$ rotation of the magnetization) repeatedly nucleating from one of the sample surfaces and subsequently traveling along the DW in the $z$ direction, leading to repeated switching of the DW internal magnetization. For samples with even larger thicknesses, a "full" $\pi$ HBL structure is observed to nucleate and propagate along the DW in the thickness direction of the sample, see Fig. 4, as well as Supplemental Material, Movie 2 [32]. There, the HBL travels along the DW in the $\pm z$ directions in a sample with $h=1.89 \mu \mathrm{m}$ subject to a field of $B_{\text {ext }}=0.6 \mathrm{mT}$. Figure 4(a) shows a HBL traveling to the $+z$ direction, a little before it punches through at the top surface. Another HBL with an opposite in-plane magnetization is nucleated shortly after the punch through and starts traveling in the $-z$ direction, i.e., towards the bottom surface [Fig. 4(b)]. After the punch through at the bottom surface and the subsequent nucleation of another HBL, the process repeats itself.

The question is now how the above observations about the internal magnetization dynamics of the DW may be used to understand the nonmonotonic $h$ dependence of $v_{\max }$ (and consequently, of $B_{\mathrm{ext}}^{\max }$ ). Theoretical calculations by Slonczewski and Malozemoff [12,20] suggest that for samples with thicknesses above the so-called Bloch line limit, i.e., for $h \gg \Lambda$, where $\Lambda=\sqrt{A / K_{\mathrm{d}}} \approx 208 \mathrm{~nm}$ is the Bloch line width parameter, the maximum velocity obeys $v_{\max }(h) \sim 1 / h$; similar behavior with $v_{\max }$ decreasing with $h$ can be seen in our results for the largest thicknesses considered.

On the other hand, for thin strips where the DW internal magnetization exhibits spatially uniform precession, one may apply the theory of Mougin et al. [33], stating that in confined geometries the Walker field assumes a modified form $H_{\mathrm{W}}=2 \pi \alpha M_{\mathrm{S}}\left|N_{x}-N_{y}\right|$, where $N_{x}$ and $N_{y}$ are the demagnetizing factors along $x$ and $y$, respectively. For the present case of PBCs along $y$, the DW length is effectively infinite, and thus $N_{y}=0$. Using the elliptic approximation for $N_{x}$ [33], one obtains $H_{\mathrm{W}}=2 \pi \alpha M_{\mathrm{S}} h /(h+\Delta)$, and $v_{\max }=$ $\left(2 \pi \gamma \Delta M_{\mathrm{S}} h\right) /(h+\Delta)$. Both of these increase with $h$ for small $h$, in qualitative agreement with our results. (a)

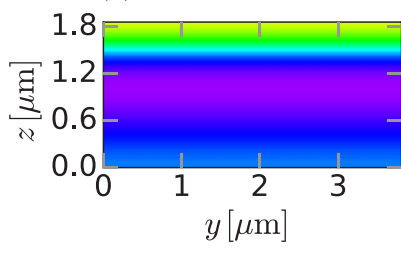

(b)

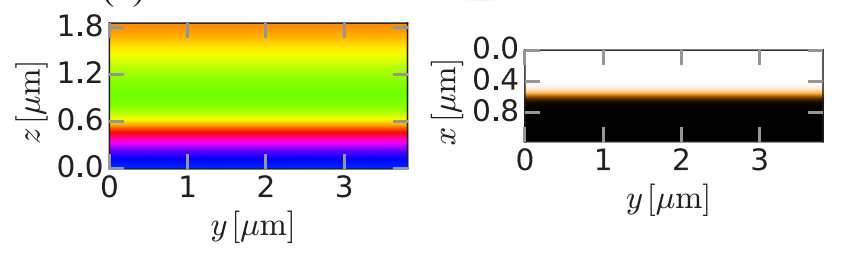

FIG. 4. A HBL subject to an applied field of $B_{\text {ext }}=0.6 \mathrm{mT}$ traveling along the DW in the $z$ direction in a strip of thickness $h=$ $1.89 \mu \mathrm{m}$ with PBCs in the $y$ direction. Left panels show snapshots of the magnetization configuration within the plane of the DW (defined as $m_{z}=0$ ), while the right panels show the corresponding magnetization that could be observed on the top surface of the sample. In (a), the HBL (with the midpoint magnetization $m_{x}^{\mathrm{BL}}=-1$, cyan color) is moving upwards (in the $+z$ direction) along the DW. Shortly after the snapshot shown in (a), it punches through the top surface. Soon after that a new HBL with opposite magnetization $\left(m_{x}^{\mathrm{BL}}=+1\right.$, red color) is nucleated and it travels to the opposite direction, i.e., in the $-z$ direction. The snapshot in (b) shows the HBL just before it punches through the bottom surface, after which the process repeats. The left panels of (a) and (b) show the magnetization in the DW plane (defined as $m_{z}=0$ ), while the right panels show the corresponding strip magnetization in the top surface of the strip. The color wheel shows the mapping between colors and the direction of the in-plane magnetization, and white and black correspond to $m_{z}=1$ and -1 , respectively.

Thus, it appears that our results for the PBC case interpolate between a small- $h$ regime where the onset of the uniform precession of the DW internal magnetization is controlled by the thickness dependent demagnetizing factor $N_{x}$ of the DW (leading to a $v_{\max }$ increasing with $h$ ), and a large- $h$ regime controlled by the nucleation and subsequent dynamics of a HBL, leading to a decreasing $v_{\max }$ with $h$ (asymptotically of the form of $\left.v_{\max } \sim 1 / h[12,20]\right)$. These two regimes are separated by a maximum of $v_{\max }$, which for our system occurs at $h \approx$ $840 \mathrm{~nm}$. For this thickness, $h$ roughly coincides with $z_{a}(h)+$ $\left[h-z_{b}(h)\right]+\pi \Lambda \approx 850 \mathrm{~nm}$ where $z_{a}(h)$ and $z_{b}(h)$ are the critical points, and $\pi \Lambda$ is the natural BL width [12]. Thus, the HBL width together with the additional thickness due to the two critical points appears to set a characteristic thickness corresponding to the maximum of the $v_{\max }(h)$ curve. In other words, as soon as the sample thickness $h$ is large enough to accommodate a full $\pi$ HBL as well as the critical points to nucleate HBLs, the energy barrier to nucleate HBLs becomes lower and the maximum achievable stable velocity $v_{\max }$ starts to decrease with increasing $h$.

\section{B. Open boundary conditions}

Next, we proceed to consider the DW dynamics in a system with OBCs, to understand the effect of the presence of strip 
edges on the onset of the excitations of internal degrees of freedom of the DW internal magnetization. The resulting DW propagation velocities $v\left(B_{\text {ext }}, h\right)$ are presented in Figs. 2(c) and 2(d). The same linear part for small fields as in the PBC case can be observed, but the velocity drop at which the linear part terminates is more abrupt and pronounced for OBCs. Also, as can be seen in Fig. 3 (green triangles), the system with $\mathrm{OBCs}$ reaches the maximum velocity for each $h$ at a lower field than the corresponding PBC system, and consequently the value of $v_{\max }$ is also lower. This difference between $\mathrm{PBCs}$ and $\mathrm{OBCs}$ is expected as the OBCs provide additional possibilities to nucleate excitations such as vertical Bloch lines from the strip edges, not present in the system with PBCs. This observation may also be rationalized within the theory of Mougin et al. [33] discussed above, by noticing that for OBCs $N_{y} \neq 0$, leading to a smaller value of $H_{W}$ (and thus of $v_{\max }$ ) as compared to the PBC case where $N_{y}=0$. Moreover, by comparing the $v\left(B_{\text {ext }}, h\right)$ data for OBCs and PBCs [Figs. 2(b) and 2(d), as well as Fig. 3], it appears that the nature of the DW dynamics becomes increasingly similar in the two cases as $h$ increases (i.e., the boundary conditions appear to become asymptotically irrelevant for large $h$ ). In particular, for the thickest system considered with $h=1.89 \mu \mathrm{m}, v_{\max }$ and $B_{\text {ext }}^{\max }$ have quite similar values for PBCs and OBCs (Fig. 3). This is understandable as based on our observations for PBCs discussed above, in thick enough samples the breakdown dynamics is expected to be dominated by nucleation of HBLs at the strip surfaces, which should not be sensitive to the boundary conditions at the strip edges.

We then proceed to consider the $h$-dependent excitations of the DW internal magnetization responsible for the velocity drop visible in Fig. 2(d), considering again the dynamics taking place for the smallest field value above $B_{\mathrm{ext}}^{\max }$ for each $h$. In thin systems $(h<210 \mathrm{~nm})$ we observe a VBL repeatedly nucleating at one of the strip edges and subsequently traveling towards the other edge [see Fig. 5(a) for a snapshot of a traveling tail-to-tail VBL in a system with $h=210 \mathrm{~nm}$; see also Supplemental Material, Movie 3]. This dynamics is similar to the dynamics found recently in simulations of thin CoPtCr samples with OBCs [13]. For somewhat thicker strips (210 $\mathrm{nm}<h<390 \mathrm{~nm}$ ) we observe a VBL nucleating from one of the strip edges and subsequently traveling back and forth between the strip edges. Moreover, in this thickness range, the VBLs start to become slightly deformed due to the flux-closing tendency at the strip surfaces: As discussed before, the surface charges due to the $\pm z$ magnetized domains result in demagnetizing fields acting on the DW magnetization and pointing along positive and negative $x$ directions at the top and bottom surfaces of the strip, respectively. These fields tend to tilt the magnetization surrounding the midpoint of the VBL away from the otherwise preferred $\pm y$ directions (i.e., from the Bloch wall structure), leading to a deformed VBL structure breaking the symmetry between the top and bottom surfaces of the strip.

Upon further increasing $h$, this VBL deformation becomes more pronounced, and their dynamics change drastically. During the propagation across the strip width along the DW, on one strip surface (face) the VBL makes almost a full $2 \pi$ rotation, while on the opposite face the magnetization rotates roughly by $\pi / 2$ only. After reaching the other strip edge, the
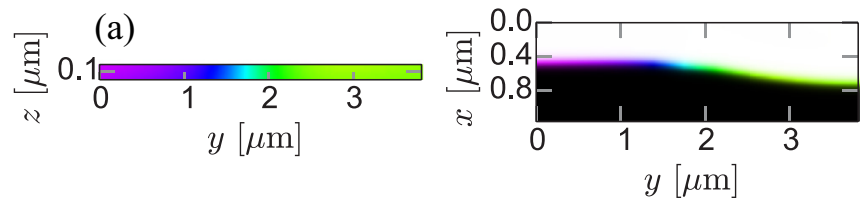

(b) $t=336.5 \mathrm{~ns}$

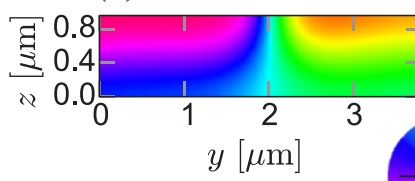

(c) $t=354.5 \mathrm{~ns}$
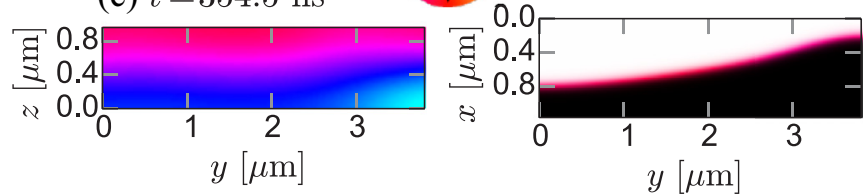

(d) $t=377.0 \mathrm{~ns}$
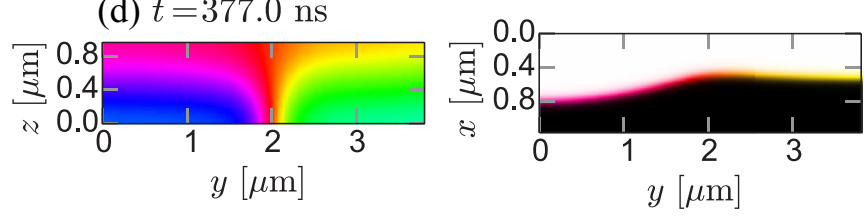

FIG. 5. VBL dynamics in systems with OBCs in the $y$ direction. Left panels show snapshots of the magnetization configuration within the plane of the DW (defined as $m_{z}=0$ ), while the right panels show the corresponding magnetization that could be observed on the top surface of the sample. (a) shows a snapshot from a strip with thickness of $h=210 \mathrm{~nm}, B_{\text {ext }}=0.32 \mathrm{mT}$, with a VBL traveling in the $+y$ direction. (b), (c), and (d) display three snapshots from a system with $h=990 \mathrm{~nm}$ and $B_{\text {ext }}=0.64 \mathrm{mT}$, with a deformed VBL with the midpoint VBL magnetization along $-x$ (cyan) propagating in the $+y$ direction in (b), followed by a transient HBL-like structure in (c). After that, as shown in (d), a deformed VBL with the midpoint magnetization along $+x$ moves in the $-y$ direction. Notice how the top view of the sample in (d) hardly contains any direct information about the presence of a VBL within the DW, apart from the retarded part at the location of the VBL. The color wheel shows the mapping between colors and the direction of the in-plane magnetization, and white and black correspond to $m_{z}=1$ and -1 , respectively.

system exhibits a short-lived transient partial HBL structure (in that the magnetization rotation is less than $\pi$ due to the limited strip thickness), followed by the propagation of a deformed VBL towards the other strip edge. Figures 5(b), 5(c), and 5(d) (and Supplemental Material, Movie 4 [32]) illustrate this in the case of a tail-to-tail VBL within a $h=990 \mathrm{~nm}$ sample; here, the head-to-head vs tail-to-tail nature of the deformed VBL may be defined by considering the magnetization profile along the DW in the middle layer of the strip, where the magnetostatic effects due to the surface charges are negligible. Figure 5(b) shows a snapshot of a deformed VBL traveling in the positive $y$ direction. The view on the top face of the strip (shown on the right) illustrates the close to $2 \pi$ rotation of the DW magnetization one would observe by just looking at the sample surface. Figure 5(c) presents a snapshot of the partial HBL structure, which then transforms into the deformed VBL traveling in the negative $y$ direction as shown in Fig. 5(d). Notice the small magnetization rotation 
$(\sim \pi / 2)$ observable on the top face of the strip [Fig. 5 (d), right panel]. The next step is the formation of another HBL-like structure (not shown), after which the process repeats itself. The circulation direction [clockwise as in Figs. 5(b), 5(c), and $5(\mathrm{~d})$, or counterclockwise] is determined by the topology of the VBL: The large spin rotation part of deformed tailto-tail VBLs exhibit clockwise rotation, while head-to-head VBLs circulate counterclockwise. In general, the propagation direction of the various Bloch line structures is determined by the direction in which the applied field tends to rotate the midpoint magnetization of the Bloch line. The full Bloch line circulation cycle in the system described in Figs. 5(b)-5(d) takes approximately $83 \mathrm{~ns}$, and the deformed VBL travels across a face of the sample in $31 \mathrm{~ns}$. Thus, the ratio of the VBL velocity to the corresponding $\mathrm{DW}$ velocity $\kappa \equiv v_{\mathrm{VBL}} / v_{\mathrm{DW}} \approx$ 8.5 , i.e., close to the value $\kappa \approx 10.2$ estimated experimentally by Thiaville et al. [23] for the material we consider here; we have measured similar $\kappa$ values varying between $8-12$ also for other sample thicknesses where VBLs occur. Dynamics of these deformed VBL structures have been previously studied for fixed film thicknesses [23,34,35], but to our knowledge the circulating VBL dynamics described above have not been reported before.

We note that when the part of the deformed VBL with more spin rotation is traveling along the bottom face of the strip [Fig. 5(d)], an experimental measurement of magnetization of the top surface of the sample would show no clear VBL structure but only a retarded part of the DW with a $\sim \pi / 2$ rotation of the DW internal magnetization along the DW. On the bottom surface the magnetization rotates again by almost $2 \pi$. The precise amount of spin rotation across the deformed VBL varies somewhat during its propagation and depends also on $h$. Observation of a retarded region within the DW may thus provide an indirect way to experimentally detect VBLs within moving DWs in thick samples in situations where the part of the VBL with more spin rotation is hiding on the bottom surface of the strip.

In strips with thicknesses $h>1.71 \mu \mathrm{m}$, the internal DW magnetization dynamics becomes similar to that in the corresponding systems with PBCs, i.e., a HBL is nucleated on one of the strip surfaces (instead of a VBL at one of the strip edges in thinner strips). However, the punch-through mechanism of the HBL is different. As a HBL approaches a strip surface, both ends of the HBL (located close to the corners of the strip) turn into structures reminiscent of the large spin rotation parts of the deformed VBLs discussed above, which then move towards each other along the surface and eventually annihilate each other. Then, another HBL is nucleated at this strip surface, and subsequently propagates towards the other surface of the strip, and the process is repeated. This is illustrated by Supplemental Material, Movie 5 [32].

$h=1.26 \mu \mathrm{m}$ to $h=1.71 \mu \mathrm{m}$ is a transition thickness region, where both of the aforementioned breakdown dynamics are seen in the same system. Within this thickness range the dynamics begin with a few HBL punch-through events, as described above. After the punch-through events the structure changes into a deformed VBL, circulating around the DW similarly as described above for "intermediate" strip thicknesses. The DW velocity $v(t)$ has two phases as well corresponding to these two different kinds of BL dynamics:
During HBL propagation the DW moves forward with a constant velocity, but during the punch through, the DW stops and travels momentarily (for a time period of roughly $\sim 1 \mathrm{~ns}$ ) backwards, and then proceeds forwards after nucleating a new HBL. In the VBL phase the domain wall is constantly moving forward with a slightly oscillating velocity, which on the average is lower than in the HBL phase. For instance, for $h=1.71 \mu \mathrm{m}$ and $B_{\text {ext }}=0.7 \mathrm{mT}$, the average DW velocity in the HBL phase is $\sim 5 \mathrm{~m} / \mathrm{s}$ higher than in the VBL phase. In Fig. 2, we always report the steady state velocity after any initial transients.

Finally, we should note that we found an anomaly at $h=1.17 \mu \mathrm{m}$ and $B_{\text {ext }}=0.7 \mathrm{mT}$ visible in Fig. 2(c) as a small island of relatively fast DW velocity as compared to the background. Inspecting the magnetization dynamics, we found that in this particular point it is similar to what is seen in the thickest systems with purely HBL-based BL dynamics with OBCs, while the surrounding region exhibits mostly VBL dynamics. This could be, e.g., due to the shortness of the simulation time, such that the system would not reach the true steady state dynamics within the simulation time. We have verified that $B_{\text {ext }}=0.7 \mathrm{mT} \pm \epsilon$, where $\epsilon=0.001 \mathrm{mT}$, results in the same behavior.

\section{DISCUSSION AND CONCLUSIONS}

Thus, we have shown that the maximum stable field-driven DW propagation velocity $v_{\max }(h)$ in garnet strips exhibits a nonmonotonic dependence on the strip thickness $h$. We identify a characteristic sample thickness resulting in the maximum of $v_{\max }(h)$ to be given roughly by the Bloch line width $\pi \Lambda$ of the material, together with the extra thickness needed to accommodate the critical points next to both strip surfaces, and describe the types of excitations (uniform magnetization rotation, nucleation of VBLs of different types, as well as HBLs) responsible for the velocity drop for thicknesses above and below the characteristic one, considering both periodic and open boundary conditions at the strip edges. We were able to qualitatively account for the observations by comparing our results with previous theoretical analysis. For thin strips, the increasing trend of $v_{\max }(h)$ with $h$ may be understood in terms of the work of Mougin et al. [33], while in the limit of thick samples our results appear to approach those of Slonczewski, obtained for thicknesses well beyond the HBL limit, $h \gg \Lambda$ [20], leading to a decreasing $v_{\max }(h)$ with $h$.

Out of the excitation modes resulting in the drop of the DW propagation velocity, the circulating motion of the high spin rotation part of a deformed (due to demagnetizing fields originating from surface charges) VBL along the perimeter of the DW in strips of intermediate thickness with OBCs, has been reported here. Experimental verification of such a mode would be an interesting avenue for future work; in the present paper, we provide some guidelines on how to achieve this. In order to observe such dynamic processes in an experiment, one obviously needs a method with a high enough spatial and temporal resolution. One such method could be time-resolved scanning transmission x-ray microscopy (STXM), which has been reported to reach subnanosecond time and $25 \mathrm{~nm}$ spatial resolution [36]. 
Finally, we point out that extending our work to the case of 3D samples with quenched disorder [37] interacting with the DW as well as with the various BL structures within the DW [13] would be another important direction for forthcoming studies. In particular, it would allow us to address the question of the possible relevance of the DW internal structure, including VBLs and HBLs, on the nature of the jerky, avalanchelike DW motion observed in the context of the Barkhausen effect [38]. Most often such dynamics have been modeled by describing the DWs as elastic interfaces in random media, with the details of the interaction kernel depending on whether the long-range dipolar interactions are thought to be relevant or not $[39,40]$. However, in such models the dynamical internal structure of the DW is neglected, and it is a pertinent question if such an approximation is valid in all cases.

\section{ACKNOWLEDGMENTS}

This work has been supported by the Academy of Finland through its Centres of Excellence Programme (2012-2017) under Project No. 251748, and an Academy Research Fellowship (L.L., Project No. 268302). We acknowledge the computational resources provided by the Aalto University School of Science "Science-IT" project, as well as those provided by CSC (Finland).
[1] G. S. Beach, C. Nistor, C. Knutson, M. Tsoi, and J. L. Erskine, Nat. Mater. 4, 741 (2005).

[2] A. Dourlat, V. Jeudy, A. Lemaître, and C. Gourdon, Phys. Rev. B 78, 161303(R) (2008).

[3] P. J. Metaxas, J. P. Jamet, A. Mougin, M. Cormier, J. Ferré, V. Baltz, B. Rodmacq, B. Dieny, and R. L. Stamps, Phys. Rev. Lett. 99, 217208 (2007).

[4] N. L. Schryer and L. R. Walker, J. Appl. Phys. 45, 5406 (1974).

[5] A. Thiaville, Y. Nakatani, J. Miltat, and Y. Suzuki, Europhys. Lett. 69, 990 (2005).

[6] T. A. Moore, I. Miron, G. Gaudin, G. Serret, S. Auffret, B. Rodmacq, A. Schuhl, S. Pizzini, J. Vogel, and M. Bonfim, Appl. Phys. Lett. 93, 262504 (2008).

[7] H. Tanigawa, K. Kondou, T. Koyama, K. Nakano, S. Kasai, N. Ohshima, S. Fukami, N. Ishiwata, and T. Ono, Appl. Phys. Express 1, 011301 (2008).

[8] S. S. Parkin, M. Hayashi, and L. Thomas, Science 320, 190 (2008).

[9] S. Fukami, T. Suzuki, N. Ohshima, K. Nagahara, and N. Ishiwata, J. Appl. Phys. 103, $07 E 718$ (2008).

[10] L. Thomas, M. Hayashi, X. Jiang, R. Moriya, C. Rettner, and S. S. Parkin, Nature (London) 443, 197 (2006).

[11] M. Yan, A. Kákay, S. Gliga, and R. Hertel, Phys. Rev. Lett. 104, 057201 (2010).

[12] A. P. Malozemoff and J. C. Slonczewski, Magnetic Domain Walls in Bubble Materials: Advances in Materials and Device Research (Academic Press, New York, 1979), Vol. 1.

[13] T. Herranen and L. Laurson, Phys. Rev. B 92, 100405 (2015).

[14] P. Bonyhard, J. Geusic, A. Bobeck, Y.-S. Chen, P. Michaelis, and J. Smith, IEEE Trans. Magn. 9, 433 (1973).

[15] J. Nielsen, S. Blank, D. Smith, G. Vella-Coleiro, F. Hagedorn, R. Barns, and W. Biolsi, J. Electron. Mater. 3, 693 (1974).

[16] A. H. Bobeck, P. I. Bonyhard, and J. E. Geusic, Proc. IEEE 63, 1176 (1975).

[17] P. Bonyhard and J. Smith, IEEE Trans. Magn. 12, 614 (1976).

[18] A. Thiaville and Y. Nakatani, in Spin Dynamics in Confined Magnetic Structures III (Springer, Berlin, Heidelberg, 2006), pp. 161-205.

[19] V. Estévez and L. Laurson, Phys. Rev. B 93, 064403 (2016).

[20] J. Slonczewski, J. Appl. Phys. 44, 1759 (1973).
[21] A. Thiaville, J. B. Youssef, Y. Nakatani, and J. Miltat, J. Appl. Phys. 69, 6090 (1991).

[22] K. Patek, A. Thiaville, and J. Miltat, Phys. Rev. B 49, 6678 (1994).

[23] A. Thiaville, J. Miltat, and J. B. Youssef, Eur. Phys. J. B 23, 37 (2001).

[24] A. Vansteenkiste, “Mumax3”, http://mumax.github.io/, v. 3.9.1.

[25] A. Vansteenkiste and B. Van de Wiele, J. Magn. Magn. Mater. 323, 2585 (2011).

[26] A. Vansteenkiste, J. Leliaert, M. Dvornik, M. Helsen, F. GarciaSanchez, and B. Van Waeyenberge, AIP Advances 4, 107133 (2014).

[27] T. L. Gilbert, IEEE Trans. Magn. 40, 3443 (2004).

[28] W. F. Brown, Micromagnetics, 18 (Interscience Publishers, New York, 1963).

[29] W. Rave, K. Ramstöck, and A. Hubert, J. Magn. Magn. Mater. 183, 329 (1998).

[30] E. Schlömann, J. Appl. Phys. 45, 369 (1974).

[31] A. Hubert and R. Schäfer, Magnetic Domains: The Analysis of Magnetic Microstructures (Springer Science \& Business Media, Berlin, Heidelberg, 1998).

[32] SeeSupplemental Material at http://link.aps.org/supplemental/ 10.1103/PhysRevB.96.144422 for movies illustrating the details of the different types of Bloch line dynamics within moving domain walls.

[33] A. Mougin, M. Cormier, J. Adam, P. Metaxas, and J. Ferré, Europhys. Lett. 78, 57007 (2007).

[34] M. Redjdal and F. Humphrey, J. Appl. Phys. 79, 6464 (1996).

[35] A. Bagneres and F. Humphrey, IEEE Trans. Magn. 28, 2344 (1992).

[36] F.-U. Stein, L. Bocklage, M. Weigand, and G. Meier, Sci. Rep. 3, 1737 (2013).

[37] J. Leliaert, B. Van de Wiele, A. Vansteenkiste, L. Laurson, G. Durin, L. Dupré, and B. Van Waeyenberge, J. Appl. Phys. 115, 17D102 (2014).

[38] G. Durin and S. Zapperi, in The Science of Hysteresis, edited by G. Bertotti and I. Mayergoyz (Academic Press, Amsterdam, 2006), Vol. II, pp. 181-267.

[39] S. Zapperi, P. Cizeau, G. Durin, and H. E. Stanley, Phys. Rev. B 58, 6353 (1998).

[40] L. Laurson, G. Durin, and S. Zapperi, Phys. Rev. B 89, 104402 (2014). 